\title{
Determination of Chlorpromazine in the Blood and Brain of Mice by High Performance Liquid Chromatography Combined with Electrochemical Detection
}

\author{
Shinji SHIBANOKI, Yukinobu GOTOH and Koichi ISHIKAWA \\ Department of Pharmacology, Nihon University School of Medicine, \\ Itabashi, Tokyo 173, Japan \\ Accepted February 29, 1984
}

\begin{abstract}
A simple and sensitive procedure was developed for determining the concentration of chlorpromazine (CPZ) in the blood and brain of mice with promethazine as the internal standard. The procedure involves (1) primary extraction by a mixture of heptane and isoamyl alcohol (99:1) in an alkaline condition and (2) determination by means of high performance liquid chromatography with electrochemical detection. The detection limit of CPZ was $0.5 \mathrm{ng}$ for one chromatographic injection. The drug was detectable in a $100 \mu \mathrm{l}$ sample of the blood by $6 \mathrm{hr}$ after an intravenous injection of $0.25 \mathrm{mg} / \mathrm{kg}$. The concentration of CPZ was about 40 times higher in the brain compared with that in the blood. The biological half life was estimated to be 87 and $65 \mathrm{~min}$, in the blood and brain, respectively. The proposed method is also applicable for the determination of other phenothiazine derivatives and effective for pharmacokinetic study of the compounds in clinics and laboratories.
\end{abstract}

Electrochemical detection has been confirmed to be a successful procedure for the determination of substances having phenolic hydroxy radicals. Many reports demonstrated the application of the detector in combination with high performance liquid chromatography for assaying monoamines in the brain (1-4), blood (5) and urine (6). Other applications of electrochemical detection have appeared for drugs which possess phenolic $\mathrm{OH}$ in their molecular structure (7-9). Heterocyclic compounds seem to react electrochemically and have been reported to be determined by the electrochemical detector $(10,11)$.

Chlorpromazine (CPZ) is a drug used in the treatment of psychotic disorders. CPZ is a strong dopamine receptor blockade and a useful drug for determining dopaminergic function in the central nervous system. Thus the intracerebral concentration of the drug gives significant information about its effects on the dopaminergic system. The conventional procedure for assaying sensitively the concentration of CPZ is gas chromatography with an electron capture detector (12), for which complex derivatization is required because of the nonvolatile property of this drug. CPZ, a phenothiazine derivative, is a heterocyclic compound and is considered to be electrochemically reactive. A report recently demonstrated the application of HPLC-ECD for determining the concentration of promethazine and other phenothiazine derivatives including CPZ in human plasma $(13,14)$. The present study shows a simplified procedure for the assay of $\mathrm{CPZ}$ in the brain and blood of mice.

\section{Materials and Methods}

Apparatus and reagents: A liquid chromatographic system (Yanagimoto L-2000, Kyoto, Japan) was used with a six port injector (Rheodyne 7.120, Berkeley, CA, U.S.A.) and a glassy carbon electrochemical detector (Yanagimoto VMD-101). The analytical column was an Ultrasphere-ODS reversedphase column (average particle size, $5 \mu \mathrm{m}$; $250 \times 4.6 \mathrm{~mm}$ i.d.; Altex Scientific, Berkeley, CA, U.S.A.). To protect the analytical column, 
a short column packed with the ODS resin $(10 \times 4.5 \mathrm{~mm}$ i.d.) was equipped. The detector potentials were set at $700 \mathrm{mv}$ versus the $\mathrm{Ag} / \mathrm{AgCl}$ reference electrode.

Cyclic voltamgraphy was performed using CV-1A (Bioanalytical Systems, West Lafayette, Indiana, U.S.A.). The working electrode was of glassy carbon and the reference electrode, $\mathrm{Ag} / \mathrm{AgCl}$. The scan rate was $100 \mathrm{mV} / \mathrm{sec}$.

CPZ hydrochloride was obtained from Shionogi Pharmaceutical Co. (Osaka, Japan). The drug was dissolved in saline immediately prior to injection. Promethazine, internal standard for the chromatography, was also purchased from Shionogi Pharmaceutical Co. Reagent grade chemicals for extraction and chromatography were obtained from a single source (Wako, Osaka, Japan) and used without further purification. The chromatographic mobile phase was a mixture of $0.1 \mathrm{M}$ acetate buffer (pH 3.5)-acetonitriltetrahydrofuran-pyridine (100:200:3:0.3).

Animals: ICR mice, each weighing about $30 \mathrm{~g}$, were used. All animals were kept in a room in which the temperature and humidity were controlled at $23 \pm 0.5^{\circ} \mathrm{C}$ and $55 \pm 5 \%$, respectively, prior to drug administration. The animals were injected intravenously with $\mathrm{CPZ}$ at dose levels of $0.1,0.5,1,2.5,5,7.5$, 10,15 and $20 \mathrm{mg} / \mathrm{kg}$ and sacrificed by decapitation $1 \mathrm{hr}$ after the administration. In another series of experiments, $10 \mathrm{mg} / \mathrm{kg}$ of the drug was intravenously injected into mice which were sacrificed 5,15 or $30 \mathrm{~min}$ or 1,2 , 3,4 or $6 \mathrm{hr}$ after the administration.

The brain was obtained as quickly as possible after sacrifice. The sample was weighed and then wrapped in aluminium foil and stored at $-80^{\circ} \mathrm{C}$ until the assay. The blood sample was collected from the carotid artery when the animal was decapitated. The whole blood was also stored at $-80^{\circ} \mathrm{C}$.

Extraction procedure: The blood sample $(100 \mu \mathrm{l})$ was transferred to a glass-stoppered tube and agitated with $100 \mu$ of distilled water containing internal standard (promethazine, $100 \mathrm{ng}$ ) and $200 \mu$ of $1 \mathrm{~N} \mathrm{NaOH}$. Five $\mathrm{ml}$ of an organic mixture of heptane: isoamyl alcohol (99:1) was added to the tube. The tube was vortexed for $1 \mathrm{~min}$ and centrifuged for $3 \mathrm{~min}$ to separate the organic layer of which $4 \mathrm{ml}$ was transferred to another tube. The tube was added with $100 \mu \mathrm{l}$ of $0.1 \mathrm{~N} \mathrm{HCl}$ and then vortexed for $1 \mathrm{~min}$. After a brief centrifugation to separate the layers, a portion of the $\mathrm{HCl}$ layer was used as the chromatographic sample.

The brain sample (about $400 \mathrm{mg}$ ) was homogenized in distilled water containing internal standard $(2,000 \mathrm{ng})$. A portion (500 $\mu \mathrm{l}$, in general) of homogenate was transferred to a glass-stoppered tube containing $500 \mu$ of $1 \mathrm{~N} \mathrm{NaOH}$. From this point, the same procedure as for the blood sample was applied. The final $\mathrm{HCl}$ layer was injected into the HPLC system.

\section{Results}

Static experiments: Hydrodynamic voltamgrams of $\mathrm{CPZ}$ and promethazine are shown in Fig. 1A. CPZ initiated the electrochemical response at $475 \mathrm{mV}$. The internal standard, promethazine, also showed the initial response at $475 \mathrm{mV}$. Both responses increased steadily to about $700 \mathrm{mV}$ and then decreased. The maximum response was higher in the internal standard than CPZ. These results agreed with those obtained by cyclic voltammetry, in which the oxidation (anodic) current appeared at about $500 \mathrm{mV}$ (Fig. 1B). The cyclic voltamgrams showed a reduction (cathodic) current of about $1 / 3$ of the oxidation phase, indicating reversible reactions of the substances.

The detector response varied directly with the amount of CPZ injected. The ratio of CPZ to the internal standard showed linear responding $(r=0.998, N=11, P<0.0001)$ in a wide range of dosages between $500 \mathrm{pg}$ and $1 \mu \mathrm{g}$ (Fig. 2). This means that a simple comparison of the peak height is adequate for the calibration of concentrations of the substance.

The recovery rate of CPZ was over $90 \%$ after adjusting for solvent loss, and the ratio of the substance to promethazine was constant in a dose range between 500 pg and $1 \mu \mathrm{g}$.

Chromatogram: In electrochemical detection, the mobile phase must be aqueous because the reaction does not occur in the organic phase. However, acetonitril, an organic solvent, has been reported to be 
used in the buffer solution for the electrochemical detector. The solvent has been utilized for shortening the retention time of hydrophobic substances in the reverse phase column. Thus the present study successfully used acetonitril for shortening and differentiating the retention times of $\mathrm{CPZ}$ and
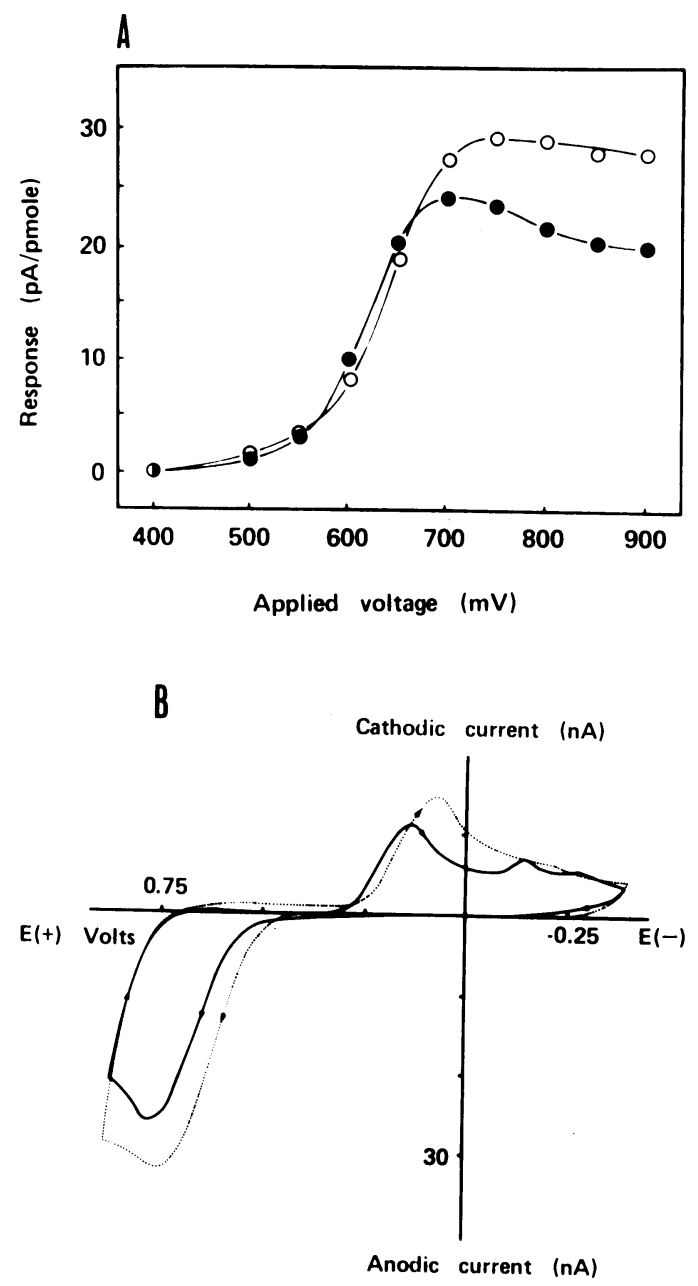

Fig. 1. Electrochemical reactability of the substances used in the present study. A. Hydrodynamic voltamgrams of $\mathrm{CPZ}(\mathbf{O})$ and promethazine $(\bigcirc)$. Detector, VMD 101 (Yanagimoto): mobile phase, $0.1 \mathrm{M}$ acetate buffer ( $\mathrm{pH}$ 3.5)-acetonitril-tetrahydrofuran-pyridine (100:200:3:0.3). B, Cyclic voltamgrams of $\mathrm{CPZ}(-)$ and promethazine $(\cdots)$. Note the similarity of both voltamgrams, demonstratirig that the cyclic voltamgram is useful for selecting the applied voltage for the electrochemical detector. promethazine. Typical chromatograms for the substances are illustrated in Fig. 3. Biogenic substances did not interfere with the quan-

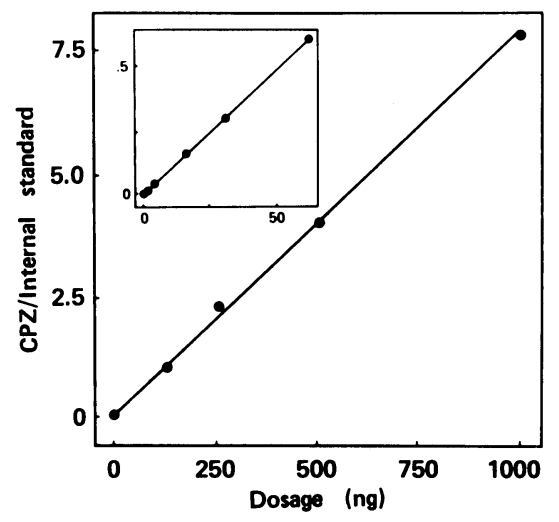

Fig. 2. Linearity of the ratio of $\mathrm{CPZ}$ and promethazine (internal standard).

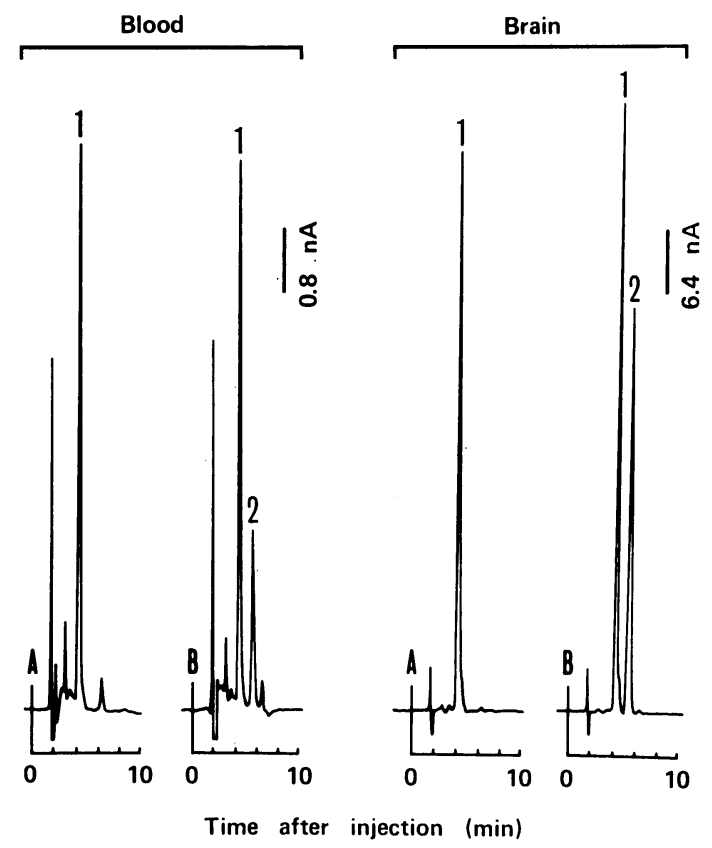

Fig. 3. Typical chromatograms of blood and brain samples. Chromatograms were obtained after a saline (A) or CPZ (B) injection. For details of chromatographic conditions, see the Methods section. Peak identities: 1, promethazine (internal standard): 2, $\mathrm{CPZ}$. Note that no biogenic substances interfered with the determination of CPZ. One run for a single assay needed about $8 \mathrm{~min}$. 
titations of $\mathrm{CPZ}$ and the internal standard in both the blood and brain. CPZ had a retention time of $5.7 \mathrm{~min}$. Assays of over 80 samples were completed in a regular $8 \mathrm{hr}$ routine workday. It may be possible to assay the drug in the blood even when it is injected intravenously at a dose level of $0.1 \mathrm{mg} / \mathrm{kg}$.

Concentrations of $\mathrm{CPZ}$ in the blood and brain: When the determination was performed 30 min after the intracerebral administration

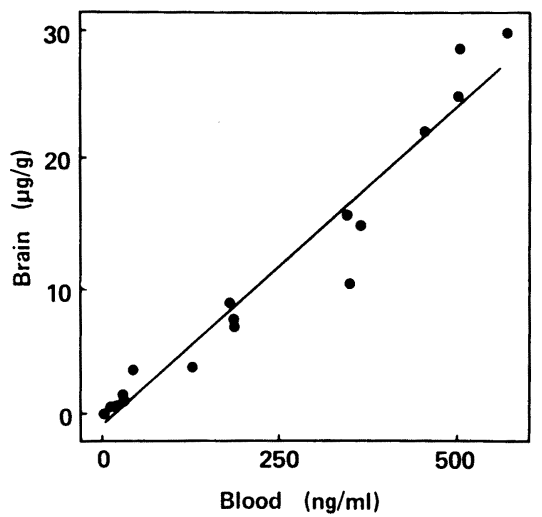

Fig. 4. Correlation of the concentration of CPZ in the blood and brain. The correlation coefficient was calculated to be 0.975 . Note that the concentrations were about 40 times higher in the brain than in the blood for all dosages tested.

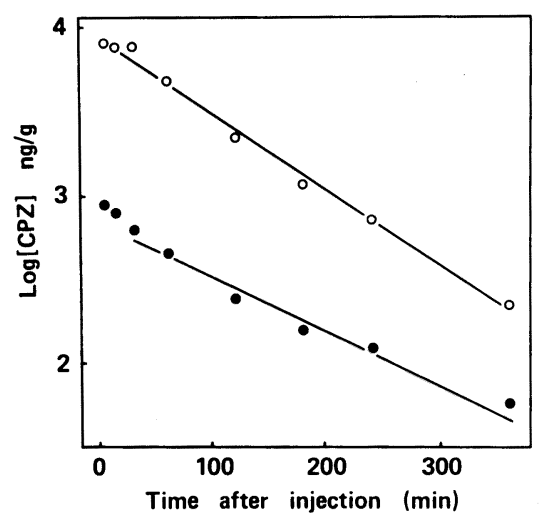

Fig. 5. Disappearance curves of CPZ from the blood (O) and brain ( $\bigcirc)$. The biological half life was estimated to be 87 and $65 \mathrm{~min}$ in the blood and brain, respectively. The regression coefficients for linearity were calculated to be -0.994 and -0.995 , respectively. of CPZ at a dose level of $0.25 \mathrm{mg} / \mathrm{kg}$, the blood concentration of the drug was estimated as $13.1 \pm 3.2 \mathrm{ng} / \mathrm{ml}$ (mean \pm S.D. of 5 determinations). In this case, the intracerebral concentration was $557.3 \pm 4.5 \mathrm{ng} / \mathrm{g}$ wet tissue, being about 40 times higher than that in the blood. This value was maintained when CPZ doses were increased by $20 \mathrm{mg} /$ $\mathrm{kg}$ (Fig. 4). The concentrations in the blood and brain correlated significantly $(r=0.975$, $\mathrm{P}<0.001$ ).

The time course of CPZ disappearance was examined after an intravenous injection of the drug at a dose level of $10 \mathrm{mg} / \mathrm{kg}$ (Fig. 5). The disappearance curves were linear both in the blood and brain with correlation coefficients of -0.994 and -0.995 , respectively $(P<0.001)$. The biological half lives of CPZ were estimated as 87 and $65 \mathrm{~min}$ for the blood and brain, respectively.

\section{Discussion}

The present study demonstrated a simple procedure for the determination of $\mathrm{CPZ}$, a neuroleptic drug. Previous reports have demonstrated the application of gas chromatography (12) or liquid chromatography combined with ultraviolet spectrometry $(15,16)$ for determination of the drug. The electrochemical reaction sufficiently occurred for the determination of $\mathrm{CPZ}$ with promethazine as the internal standard, and the proposed HPLC-ECD method was more sensitive than those conventional approaches. The heterocyclic compound has a long retention time on a reverse phase column, such as ODS in the present study, because of its physicochemical properties. If the mobile phase used for routine assay of monoamines, a citrate buffer containing tetrahydrofuran (4), was applied, the retention time was about $4 \mathrm{hr}$ for CPZ. This means that the mobile phase must include a stronger organic component. A high concentration of organic solvents is not, in general, applicable to the electrochemical detector because the reaction occurs in only aqueous states. Acetonitril is an organic solvent which is utilized as a medium for promoting reactions involving ionization. A previous report utilized, indeed, successfully acetonitril in electrochemical detection (13). This fact prompted us to 
choose the solvent in the chromatographic system in the present study. Finally, 66\% of acetonitril was found to be the most efficient for separating $C P Z$ and promethazine, the internal standard, with a citrate buffer $(\mathrm{pH}$ 3.5 ). No biogenic substances interfered with the determination of both CPZ and internal standard under the chromatographic conditions cited in the Methods section.

Electrode responses depend upon the applied voltage. Although increases in the applied voltages result in more responses, the increases are accompanied by increases in background and noise currents which interfere with suitable determination of the substances $(1,6)$. This means that the voltage has to be selected for CPZ and the internal standard. Both substances showed similar electrochemical behavior with the maximum response occurring at about 700 $\mathrm{mV}$. The electrochemical reaction reportedly occurs at the hydroxy group on the benzene ring (phenolic $\mathrm{OH}$ ). However, the present substances showed the reaction, even though they have no such moiety. The electrochemical reaction is, therefore, considered to be induced not only on phenolic $\mathrm{OH}$ but also on another site in a molecular structure. This fact extends the application of electrochemical detection. Cyclic voltammetry is the simple approach to demonstrate whether a substance is detectable by HPLCECD or not and to decide the suitable applied voltage for the determination.

Both CPZ and promethazine showed linear responses in a wide range of doses between $500 \mathrm{pg}$ and $1 \mu \mathrm{g}$. This resulted in a linearity of the ratio of the drug to internal standard. All quantitations were therefore based on peak heights of the resulting chromatogram. Ratios of the peak heights for the substances and the internal standard were compared for samples and standards taken through the entire extraction procedure, as described previously (4). The procedure also provided a treatment to include the recovery rate in the estimation of tissue concentration. The recovery rate was also constant for both substances in the wide range tested, showing values over $90 \%$ after adjusting for solvent loss. The overall precision of the present procedure was found to be less than $\pm 3.7 \%$ standard deviation for the drug.

The drug was detectable in the blood after an intravenous injection of $0.25 \mathrm{mg} / \mathrm{kg}$. While a recent report demonstrated the application of HPLC-ECD for the determination of phenothiazines in human plasma (14), the present modification yielded a higher sensitivity. The dose administered in the present study was about 10 to 40 times less than routine doses administered in clinics. Furthermore, the sample volume required for the assay was $100 \mu \mathrm{l}$. This implies that the present procedure can be applied for clinical laboratories as well as for pharmacological research.

The intracerebral concentration of $\mathrm{CPZ}$ was 40 times higher than that in the blood. The ratio was very high in comparison with that of, for example, morphine, of which the concentration was 10 times less in the brain than in the blood (9). This means that the drug penetrates into the brain easily from the blood. When CPZ was injected at a dose level of $10 \mathrm{mg} / \mathrm{kg}$, it was possible to monitor its concentration in the blood with a $100 \mu \mathrm{l}$ sample by $24 \mathrm{hr}$ after the administration. This fact is useful for pharmacokinetic experiments with small laboratory animals.

In conclusion, HPLC-ECD is a sensitive procedure for determining the pharmacokinetics of CPZ, a phenothiazine derivative, in both clinical and laboratory experiments. The method may also applicable for other heterocyclic compounds as well as for phenothiazines.

\section{References}

1 Refshauge, C., Kissinger, P.T., Dreiling, C.L., Blank, C.L., Freeman, R. and Adams, R.N.: New high performance liquid chromatographic analysis of brain catecholamines. Life Sci. 14, 311-322 (1974)

2 Kempf, E. and Mandel, P.: Reverse-phase highperformance liquid chromatographic separation and electrochemical detection of norepinephrine, dopamine, serotonin, and related major metabolites. Anal. Biochem. 112, 223-231 (1981)

3 Sperk, G.: Simultaneous determination of serotonin, 5-hydroxyindoleacetic acid, 3,4dihydroxyphenylacetic acid and homovanillic acid by high performance liquid chromatography with electrochemical detection. J. Neurochem. 
38, 840-843 (1982)

4 Ishikawa, K. and McGaugh, J.L.: Simultaneous determiriation of monoamine transmitters, precursors and metabolites in a single mouse brain. J. Chromatogr. 229, 35-46 (1982)

5 Watson, E.: Liquid chromatography with electrochemical detection for plasma norepinephrine and epinephrine. Life Sci. 28, 493-497 (1981)

6 Goto, M., Nakamura, T. and Ishii, D.: Microhigh performance liquid chromatographic system with micro precolumn and dual electrochemical detector for direct injection analysis of catecholamines in body fluid. J. Chromatogr. 226, 33-42 (1981)

7 Riggin, R.M. and Kissinger, P.T.: Determination of tetrahydroisoquinoline alkaloids in biological materials with high performance liquid chromatography. Anal. Chem. 49, 530-533 (1977)

8 Oosterhuis, B. and Boxtel, C.J.: Determination of salbutamol in human plasma with bimodal high-performance liquid chromatography and rotated disc amperometric detector. J. Chromatogr. 232, 327-334 (1982)

9 Ishikawa, K., McGaugh, J.L., Shibanoki, S. and Kubo, T.: A sensitive procedure for determination of morphine in mouse whole blood by high performance liquid chromatography with electrochemical detection. Japan. J. Pharmacol. 32, 969-971 (1982)

10 Akpofure, C., Riley, C.A., Sinkule, J.A. and Evans, W.E.: Quantitation of daunorubicin and its metabolites by high-performance liquid chromatography with electrochemical detection.
J. Chromatogr. 232, 377-383 (1982)

11 Prescott, W.R., Jr., Boyd, B.K. and Seaton, J.F.: High-performance liquid chromatographic separation of the two estrogen isomers of estradiol with electrochemical detection. J. Chromatogr. 234, 513-516 (1982)

12 Noonan, J.S., Blake, J.W., Murdick, P.W. and Ray, R.S.: Bromination of phenothiazine tranquilizers: A method for sensitive and specific detection. Life Sci. 11, 363-373 (1972)

13 Wallace, J.E., Shimek, E.L., Jr., Stavchancky, S. and Harris, S.C.: Determination of promethazine and other phenothiazine compounds by liquid chromatography with electrochemical detection. Anal. Chem. 53, 960-962 (1981)

14 Murakami, K., Ueno, T., Hijikata, J., Shirasawa, K. and Muto, T.: Simultaneous determination of chlorpromazine and levomepromazine in human plasma and urine by high-performance liquid chromatography using electrochemical detection. J. Chromatogr. 227, 103-112 (1982)

15 Wheals, B.B.: Simple preparation of a bonded cation-exchange packing material and its application to the separation of phenothiazines by high-performance liquid chromatography. J. Chromatogr. 177, 263-270 (1979)

16 Greving, J.E., Bouman. H., Jonkman, J.H.G., Westenberg, H.G.M. and De Zeeuw, R.A.: Analysis of quaternary ammonium compounds and basic drugs based on ion-pair adsorption high-performance liquid chromatography. J. Chromatogr. 186, 683-690 (1979) 